\title{
Attentional modulation of desensitization to odor
}

\author{
Nicholas Fallon ${ }^{1}$ [D $\cdot$ Timo Giesbrecht ${ }^{2} \cdot$ Andrej Stancak $^{1}$
}

Published online: 22 May 2018

(C) The Author(s) 2018

\begin{abstract}
Subjective and behavioral responsiveness to odor diminishes during prolonged exposure. The precise mechanisms underlying olfactory desensitization are not fully understood, but previous studies indicate that the phenomenon may be modulated by central-cognitive processes. The present study investigated the effect of attention on perceived intensity during exposure to a pleasant odor. A within-subjects design was utilized with 19 participants attending 2 sessions. During each session, participants continuously rated their perceived intensity of a 10-minute exposure to a pleasant fragrance administered using an olfactometer. An auditory oddball task was implemented to manipulate the focus of attention in each session. Participants were instructed to either direct their attention toward the sounds, but still to rate odor, or to focus entirely on rating the odor. Analysis revealed three 50 -second time windows with significantly lower mean intensity ratings during the distraction condition. Curve fitting of the data disclosed a linear function of desensitization in the focused attention condition compared with an exponential decay function during distraction condition, indicating an increased rate of initial desensitization when attention is distracted away from the odor. In the focused-attention condition, perceived intensity demonstrated a regular pattern of odor sensitivity occurring at approximately 1-2 minutes intervals following initial desensitization. Spectral analysis of low-frequency oscillations confirmed the presence of augmented spectral power in this frequency range during focused relative to distracted conditions. The findings demonstrate for the first time modulation of odor desensitization specifically by attentional factors, exemplifying the relevance of top-down control for ongoing perception of odor.
\end{abstract}

Keywords Olfaction · Adaptation and aftereffects $\cdot$ Cognitive and attentional control

Psychophysical studies have shown that perceived odor intensity diminishes during the course of prolonged or repetitive olfactory stimuli (Berglund, 1974; Cain, 1969; Ekman, Berglund, Berglund, \& Lindvall, 1967). It is generally accepted that a reduction of perceived odor intensity occurs concurrently at both peripheral and central levels. Peripheral reductions in responsiveness that occur at the level of olfactory receptor neurons (Hummel, Knecht, \& Kobal, 1996;

Electronic supplementary material The online version of this article (https://doi.org/10.3758/s13414-018-1539-2) contains supplementary material, which is available to authorized users.

Nicholas Fallon

nickfal@liverpool.ac.uk

1 Department of Psychological Sciences, Institute of Psychology, Health, and Society, University of Liverpool, Eleanor Rathbone Building, Bedford Street South, Liverpool L69 7ZA, UK

2 Unilever Research \& Development, Port Sunlight, UK
Kurahashi \& Menini, 1997) are often referred to as adaptation, whereas central-cognitive processes (such as changes in brain activity or reduced behavioral responsiveness) may be termed habituation (Dalton, 2000; Thompson \& Spencer, 1966). Eletro-olfactogram recordings directly from olfactory receptors demonstrated that behavioral desensitization is independent from adaptation in the periphery (Hummel et al., 1996), and functional magnetic resonance imaging (fMRI) studies revealed blood-oxygen-level-dependent (BOLD) signal changes associated with desensitization to odor stimuli that were predominantly encoded in primary olfactory cortices (Poellinger et al., 2001). However, despite this functional and semantic demarcation, it is clear that central and peripheral processes occur in parallel and share a complex relationship (Pellegrino, Sinding, de Wijk, \& Hummel, 2017), and it is their cumulative effects that determine subjective changes in perception (Dalton, 2000). This holistic decrease in perceived odor intensity can be referred to as olfactory desensitization (Stuck, Fadel, Hummel, \& Sommer, 2014). Further investigation of peripheral and central-cognitive processes in isolation 
is critical to improving our understanding of the mechanisms underlying olfactory desensitization.

One way to investigate central-cognitive aspects of desensitization in isolation is via novel experimental paradigms that target central processing. Attention can be directed toward olfaction in a similar fashion to other sensory modalities (Keller, 2011), and the composition of perceived "odor objects" can be influenced by top-down processes (Wilson $\&$ Sullivan, 2011). Previous research has demonstrated that allocation of attention toward stimuli reduces response times for odor perception (Spence, Kettenmann, Kobal, \& McGlone, 2000, 2001; Spence, McGlone, Kettenmann, \& Kobal, 2001) and also modulates perceptions of odor intensity (Rolls, Grabenhorst, Margot, da Silva, \& Velazco, 2008; Spence, McGlone, et al., 2001). Electroencephalographic (EEG) studies have shown that focused attention increases olfactory event-related potentials (Geisler \& Murphy, 2000; Krauel, Pause, Sojka, Schott, \& Ferstl, 1998; Masago, Shimomura, Iwanaga, \& Katsuura, 2001; Pause, Sojka, \& Ferstl, 1997), and fMRI studies report variations in hemodynamic brain responses when attention is focused toward an odor (Plailly, Howard, Gitelman, \& Gottfried, 2008; Sabri, Radnovich, Li, \& Kareken, 2005; Veldhuizen \& Small, 2011; Zelano et al., 2005) or toward a particular quality of an odor (Rolls et al., 2008). Moreover, research indicates that the process of olfactory desensitization may be influenced by varying top-down factors such as odor hedonics (Jacob, Fraser, Wang, Walker, \& O'Connor, 2003), prior experience (Smeets \& Dalton, 2002; Wysocki, Dalton, Brody, \& Lawley, 1997), or cognitive manipulations of odor salience (Dalton, 1996; Dalton, Wysocki, Brody, \& Lawley, 1997; Kobayashi et al., 2008; Smeets \& Dalton, 2005).

Despite the previous research reviewed above, to our knowledge no study has investigated how focus of attention itself may affect the process of olfactory desensitization. In the present work, we investigated the effects of attention on perceived intensity of a pleasant odor that was presented for a 10 minute period to induce desensitization. The stimulus was presented continuously and controlled using an olfactometer with constant odor concentration and flow rate to minimize variation in the periphery. A within-subjects design was utilized, and an auditory oddball task was employed to manipulate focus of attention during the period of olfactory desensitization.

\section{Materials and methods}

\section{Participants}

Nineteen participants (seven males) ages $26.8 \pm 3.0$ years ( $M \pm$ $S D$ ) took part in the study. Participants were recruited through campus advertisement at the University of Liverpool.
Informed consent was obtained from all participants in accordance with the Declaration of Helsinki, and the study was approved by the University Research Ethics Committee at the University of Liverpool. Participants between 18 and 35 years of age were considered for participation, and volunteers taking regular medication or those suffering from respiratory, neurological, or olfactory disease or disorders were excluded. The number of participants was based on previous psychophysical studies of prolonged odor exposures (Stuck et al., 2014) or investigations of effects of attention on olfaction (Spence, McGlone, et al., 2001).

Eligibility and sense of smell was assessed prior to the experiment using the identification test from the Sniffin' Sticks odor test battery (Hummel, Sekinger, Wolf, Pauli, \& Kobal, 1997). Participants were asked to identify 12 odors from four visually presented options, and a minimum score of 9 correct probes was required for inclusion in the study. Participants were scheduled to attend two experimental sessions 1 week apart: In one session they focused on the odor (focused attention condition), and in another their focus was distracted away from the olfactory stimulus by means of an auditory oddball task (distraction condition). The order of conditions was balanced across participants. All volunteers were compensated for time and travel expenses following completion of the study.

\section{Odor stimuli}

The odor utilized for the study was Snow Queen 10, a pleasant floral, green fragrance with minimal trigeminal activity according to component ingredients and profile (UR224459/00, Givaudin Ltd., Switzerland). For the purpose of the experiment a $0.1 \%(\mathrm{v} / \mathrm{v})$ dilution of fragrance in propylene glycol (1,2-Propanediol 99\%, Sigma-Aldrich Co., USA) was prepared. For the clean-air control stimulus, pure propylene glycol solution was used. The olfactometer utilized was a custom-made flow olfactometer, with eight individual valves allowing for variable flow rates and a carbon filtered air intake (OL-2, Dancer Design Ltd., UK). The olfactometer delivered odors via two polytetrafluoroethylene tubes of 2-mm diameter ending approximately $2 \mathrm{~cm}$ below the nostrils. The flow rate was set to 2.2 liters/minute at the beginning of each experimental session and exhibited minimal variation $(<0.1 \mathrm{l} /$ min) throughout the course of exposure to ensure odor concentration was maintained. Delivery of the odor was preceded and succeeded by clean air at a matching flow rate to avoid sudden increases in airflow associated with presentation of an odor. Before and after each experiment, the ambient air in the chamber was cleansed of residual odors using a carbon filtered Blueair 203 Heppasilent Particle Filter system (Blueair AB, Sweden). 


\section{Procedure}

Participants were tested in a sound-attenuated chamber, and clean, carbon-filtered air was pumped from outside the room, through the olfactometer, to the nosepiece. A PneumoTrace II Piezo-electric transducer was fitted around the torso at the level of the epigastrium to record respiratory movements (AD Instruments Pty Ltd., Australia). Participants were seated approximately $0.7 \mathrm{~m}$ from a 19 -inch monitor. Prior to the experiment, each participant rated odor stimuli for intensity, pleasantness, and familiarity using visual analogue scales (VAS). For intensity ratings, the VAS anchors were not perceivable to extremely Intense; for pleasantness, extremely unpleasant to extremely pleasant; and for familiarity, extremely unfamiliar to extremely familiar.

After initial evaluations of the odor, participants were provided with a response meter (AD Instruments Pty Ltd., Australia) with a slide-bar control that allowed them to continuously quantify their response to the stimulus. They were instructed that the slide bar was for continuous rating of the intensity of the odor and underwent brief training for suitable use. The anchors on the slide bar ranged from not perceivable to extremely intense. All participants were instructed that odors may or may not be presented at any time during the session and that they should try to be as fast and accurate as possible with their continuous ratings.

During all experimental sessions, the odor presentation was accompanied by an auditory oddball task. Auditory beep tones (total $=180)$ were presented at $60-\mathrm{dB}$ sound pressure level using stereophonic speakers positioned $0.8 \mathrm{~m}$ from the subject. Stimuli were presented at pseudorandom intervals (range 3-5 s, mean interstimulus interval $4 \mathrm{~s}$ ), and thirty six tones (20\%, interspersed randomly throughout the session) were presented at a noticeably higher pitch $(800 \mathrm{~Hz})$ to the majority $(500 \mathrm{~Hz})$. In the distraction condition, participants were instructed to maintain a running count of the total number of "odd" beeps (those with a higher pitch). They were informed that their final count would be required at the completion of the session, but that continuous rating of odor intensity using the response meter was still a necessary task. In the focusedattention condition, participants were informed that they would hear a series of tones during the experiment, but that they should ignore them and focus entirely on rating any odor that they may detect. The order of conditions was counterbalanced across sessions.

In both conditions, the experimental paradigm encompassed a 60-s presentation of clean air, followed by a 600 -s presentation of the pleasant odor, followed by a 60 -s presentation of clean air (flow rate $2.21 / \mathrm{min}$ ). During the experiment, the monitor displayed basic instructions, reminding participants to relax and breathe normally through their nose, to rate the intensity of any odor they may perceive, and to either IGNORE the beeps or FOCUS on counting the odd beeps. Auditory and odor stimuli were administered using Cogent 2000 software in MATLAB 7.13 (The MathWorks Inc., Natick, MA, USA).

\section{Data acquisition}

Data were recorded using an AD Instruments PowerLab 8/35 system (AD Instruments Ltd., Australia) and LabChart 7.3 acquisition software (AD Instruments Ltd., Australia) at a sampling rate of $1000 \mathrm{~Hz}$.

\section{Analysis}

Raw data for subjective ratings of intensity were exported to MATLAB 7.13 (The MathWorks Inc., Natick, MA, USA) and resampled to $1 \mathrm{~Hz}$, resulting in 720 samples. The maximum perceived odor intensity during the first 10 seconds of odor presentation was exported for each individual in both conditions, and a within-subjects $t$ test was utilized to compare maximum perceived odor intensity between conditions. A within-subjects $t$ test was performed to compare mean intensity ratings at each of the 720 samples in the experiment, and permutation analysis (2,000 permutations) was employed to correct for the large number of tests required (Maris \& Oostenveld, 2007). Time periods exhibiting consistent differences in mean intensity ratings were identified, and the average intensity rating during each epoch was determined. A within-subjects $t$ test, or Wilcoxon signed-rank test (depending on distribution of data), was performed to compare mean intensity ratings across both conditions in each of these time periods of interest.

\section{Curve-fitting analysis}

To further compare the mean rate of habituation during distraction and focused attention conditions, mean data from the time point of the maximum perceived odor intensity until 120 seconds later (representing the period of desensitization from maximum to minimum intensity ratings) was determined for distraction and focused attention data sets. The curve fitting toolbox in MATLAB (The MathWorks Inc., Natick, MA, USA), was employed to identify the mathematical function that best fitted the group data set for each condition.

\section{Spectral analysis}

To evaluate possible low-frequency oscillatory changes in perceived odor intensity during focused attention and distraction conditions, perceived intensity data for the period $120 \mathrm{~s}$ to 660 $\mathrm{s}$ following onset of odor was exported for each subject. This time window covered the period following the rapid initial desensitization (which was investigated using the previously described curve-analysis method). The relative power spectral 
density was estimated using Welch's averaged modified periodogram method. This yielded a power spectral density ranging from 0 to $0.5 \mathrm{~Hz}$ (129 components) for each subject and condition. A within-subjects $t$ test was utilized to compare mean intensity ratings at each of 129 frequency components, and permutation analysis (2,000 permutations) employed to correct for multiple tests.

\section{Respiratory data analysis}

The respiratory movement signals were resampled to $10 \mathrm{~Hz}$, and inspiratory peaks occurring in each 10 -min recording were identified. Respiration rate (cycles/minute) was evaluated for time periods showing significant differences in intensity ratings between conditions, and mean respiration rates during these periods were compared between conditions using a within-subjects $t$ test. In addition, we calculated mean respiration rates for the period of exposure for each subject using a 60 -s moving window average (which necessitated the removal of $30 \mathrm{~s}$ of data at the beginning and end of time series). Pearson's correlation coefficients for individual respiration rate and intensity ratings were calculated for each condition. One-sample $t$ tests were utilized to investigate whether individual correlations between respiration rate and odor perception across the group significantly differed from the null, and a paired-samples $t$ test was utilized to evaluate differences between conditions.

\section{Results}

\section{Pretest VAS odor ratings}

Within-subjects $t$ tests revealed no significant differences between VAS scores for perceived odor intensities prior to the focused attention (5.29 $\pm 1.66, M \pm S D)$, and distraction (4.73 \pm 1.71 ) conditions; $t(18)=1.77, p=.09$, or for perceived pleasantness in focused attention $(6.81 \pm 0.92)$ and distraction $(6.98 \pm 1.01)$ conditions; $t(18)=-0.51, p=.62$.

\section{In-test intensity ratings}

Mean continuous intensity ratings for both conditions (see Fig. 1) exhibit a steep incline immediately following odor onset and achieve maximal perception within $10 \mathrm{~s}$. This was followed by a gradual decline in perceived intensity which lasted approximately $120 \mathrm{~s}$ to minimal perception. The maximum odor intensity perceived during the first 10 seconds of odor presentation was determined for each subject in both conditions. A within-subjects $t$ test revealed no significant difference between maximum perceived odor intensities for the focused attention $(4.85 \pm 1.48)$ and distraction conditions $(4.32 \pm 2.11) ; t(18)=1.13, p=.22$. The continuous intensity ratings for each participant in both conditions are illustrated in Supplementary Fig. S1.

Permutation analysis identified three time periods when mean intensity ratings differed significantly between conditions (see Fig. 1, gray rectangles). The first significant difference between focused and distraction conditions occurred during the initial period of pronounced desensitization. Later on in the exposure, recurrent phases of significantly increased odor-intensity perception in ratings were evident in the focused-attention condition relative to the distraction condition. The pattern of recurrent-odor perception in the focusedattention condition displayed a quasi-oscillatory profile, which was dominated by low-frequency oscillations lasting in the region of $100 \mathrm{~s}$.

For each time window highlighted by permutation analyses, mean intensity ratings were exported, and a comparison test was performed. At 15 to $65 \mathrm{~s}$ following onset of odor stimuli, a significant difference was seen between mean intensity ratings for the focused attention $(3.12 \pm 1.83, M \pm S D)$ and distraction conditions $(2.02 \pm 1.49) ; t(18)=2.28, p=.035$. Between 290 and $340 \mathrm{~s}$ following onset of odor stimuli, a Wilcoxon signed-rank test showed that intensity ratings for the focused attention condition were significantly greater than during the distraction condition; $z=-1.97, p=.049$. Likewise, between 420 and $470 \mathrm{~s}$ following onset of odor stimuli, mean intensity ratings for the focused-attention condition were greater than those during the distraction condition; $z=$ $-2.01, p=.044$.

\section{Curve-fitting analysis of initial desensitization}

Mean perceived intensity data from the time point of the maximum perceived odor intensity until 120 seconds later (minimal odor perception) was exported for both conditions. The curve function that best explained the data (highest adjusted $R^{2}$ value, lowest sum of squared error value) was fitted for each condition (see Fig. 2) in the MATLAB curve-fitting toolbox. A linear polynomial function best described the curve in the focused-attention condition (adjusted $R^{2}=0.98, S S E=$ 1.87), whereas a second-order exponential decay function demonstrated the best fit in the distraction condition (adjusted $R^{2}=0.97, S S E=1.31$ ). The greatest difference in curve functions covers a similar time period to the first time window, which showed a significant difference in mean intensity ratings. This finding suggests that the steeper exponential rate of desensitization during the distraction condition may account for reduced intensity ratings seen during the time period from 15 to 65 seconds following onset of odor presentation.

\section{Recurrent perceived odor intensity}

To evaluate any potential spectral pattern explaining later differences in odor perception, power spectral density 


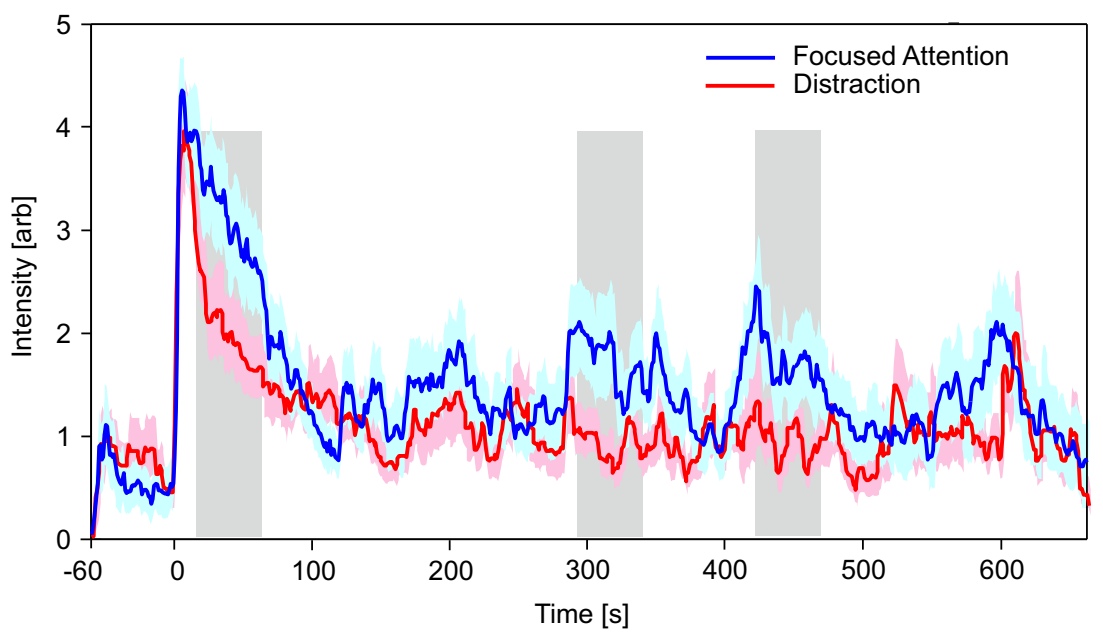

Fig. 1 Mean perceived intensity ratings and desensitization curves. Blue indicates mean intensity ratings (arbitrary units) with shading to indicate standard error distribution for all 720 timepoints in the focused attention

from 0 to $0.5 \mathrm{~Hz}$ (129 points) was computed for each subject and condition in the period of 120 to $660 \mathrm{~s}$ following onset of odor. This covers the residual exposure following the period of initial desensitization. A withinsubjects $t$ revealed larger log-transformed absolute spectral power for continuous intensity ratings in the focusedattention, relative to distraction, condition. This effect occurred in a frequency window ranging from 0.008 to $0.015 \mathrm{~Hz}$, indicative of slow oscillatory activity with a recurring profile of between 65 and $125 \mathrm{~s} ; t(18)=2.47$, $p=.025$ (see Fig. 3).

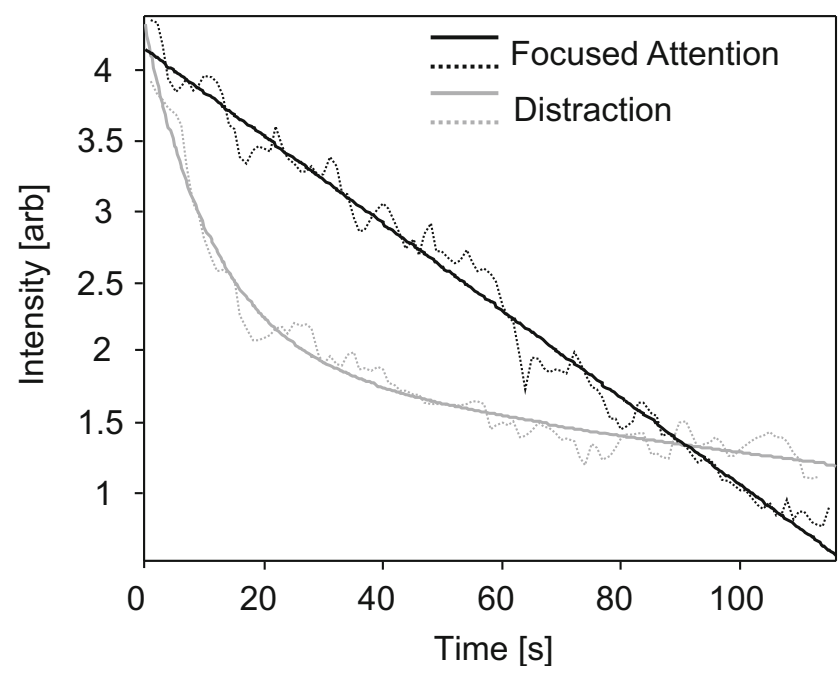

Fig. 2 Desensitization data and curve functions of best fit. Black dashed and solid lines show mean intensity ratings (arbitrary units) and curve fitting, respectively, for 120 seconds following maximum perceived intensity in the focused attention condition; gray solid and dashed lines represent data and curve fitting for the distraction condition. Note the divergence between curves in the period 10-80 seconds following maximum perceived odor intensity. condition; red indicates the distraction condition. Gray rectangles indicate time periods when mean intensity ratings differ significantly between conditions. (Color figure online)

\section{Respiratory data}

In the time periods $15-65 \mathrm{~s}$ following stimulus onset, a within-subjects $t$ test revealed no significant difference in respiration rate in the focused-attention $(13.39 \pm 2.69)$ and distraction (13.64 \pm 3.69$)$ conditions; $t(16)=-0.38, p=.71$. Similarly, no difference was observed during the time periods $350-400 \mathrm{~s}$ for the focus $(14.02 \pm 2.77)$ and distraction $(13.70$ \pm 3.23 ) conditions; $t(16)=-0.34, p=.74$, or during the period 480 to $530 \mathrm{~s}$ after stimulus onset for the focus $(13.70 \pm 2.47)$ and distraction $(14.40 \pm 2.12)$ conditions; $t(16)=-1.13, p=$ .27. One-sample $t$ tests to compare correlation coefficients for individual correlations between respiration rate and odor perception in the focused-attention condition did not indicate a significant relationship $(p>.05)$. However, there was a borderline positive relationship between respiration rate and odor perception, $\mathrm{t}(18)=2.03, p=.057$, in the distracted condition. Furthermore, a paired-samples $t$ test revealed a significant difference between correlation coefficients between conditions, $\mathrm{t}(18)=-2.36, p=.03$, suggesting that respiration rate is more closely related to odor perception in distracted, relative to focused, conditions.

\section{Discussion}

The findings demonstrate the effects of focused attention on odor desensitization, comprising a slower and linear decrease in perceived odor intensity during the first minute of odor exposure when attention was focused toward the odor, compared with a steeper, exponential decay seen in the distraction condition. Furthermore, odor desensitization was later interrupted by two recurrent periods of increased odor sensitivity highlighted by significant differences in low-frequency 


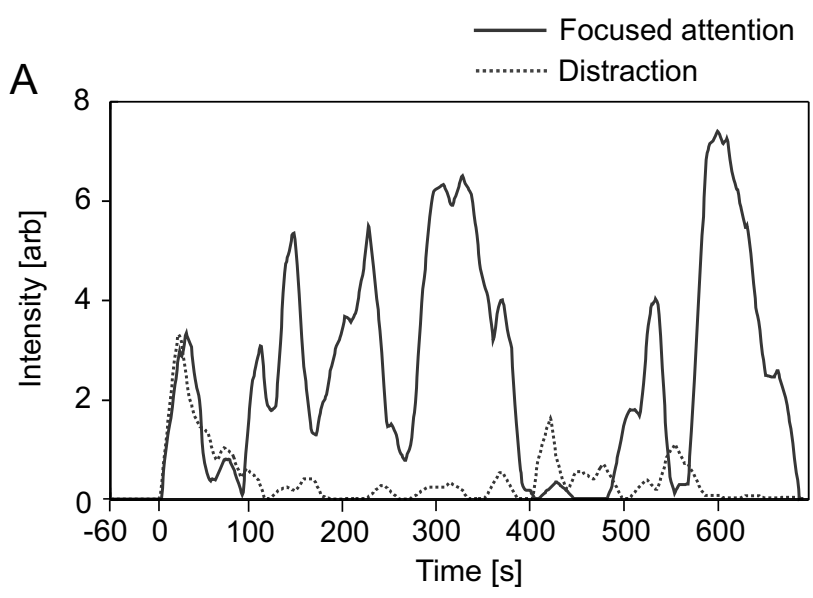

B
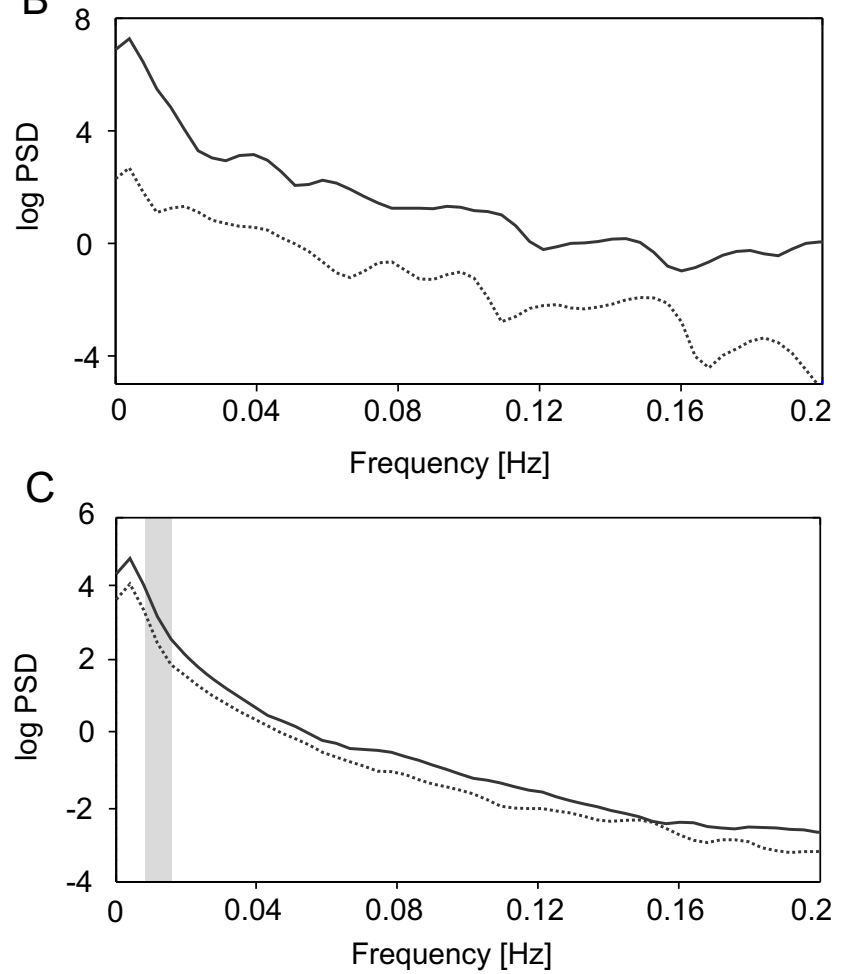

Fig. 3 a Perceived odor intensity ratings for a single subject in focused attention (solid line) and distraction conditions (dashed line). b Log power spectral density for slow oscillations $(<0.2 \mathrm{~Hz})$ in same single subject for focused attention (solid) and distraction (dashed) conditions. c Log power spectral density for all subjects for focused attention (solid) and distraction (dashed) conditions. Gray rectangle indicates frequencies demonstrating a significant difference in relative power between conditions

oscillations of subjective ratings during the focused-attention, relative to distraction, condition.

The initial, rapid decrease in odor sensitivity during the first minute of exposure was augmented in the distraction condition, which accords with previous findings indicating a significant effect of context on desensitization, particularly in the minute immediately following onset of an odor (Kobayashi et al., 2008). The pattern seen in the present study also reflects the temporal activation pattern seen in primary olfactory cortices using fMRI, manifesting a sharp decrease in activation within 1 minute of odor exposure (Sobel et al., 2000). Thus, focused attention can be seen to decrease the rate of initial desensitization seen in the period immediately following odor presentation. Previous studies indicated reduced peak intensity perception during prolonged odor perceptions in distracted conditions (Hoffmann-Hensel, Sijben, RodriguezRaecke, \& Freiherr, 2017). While our data revealed a trend toward decreased peak odor perception in the distracted condition, this did not achieve statistical significance, which may be due to single trials necessitated by the prolonged nature of exposure in our study. The finding of recurrent periods of odor sensitivity in the focused-attention condition during the latency periods $290-350 \mathrm{~s}$ and $420-470 \mathrm{~s}$ are novel to this study. However, visual examination of data from previous research suggests that such fluctuating oscillatory profiles of odor perception during prolonged odor desensitization may also be evident in earlier studies (Dalton, 1996; Dalton et al., 1997; Kobayashi et al., 2008), although any such phenomenon has so far remained unreported. Despite using comparatively shorter odor stimuli, two brain imaging studies (Poellinger et al., 2001; Sobel et al., 2000) have also shown recurrent increases in activation of olfactory regions following the initial rapid decrease seen during desensitization. This pattern may indicate a mechanism that governs the process of switching between olfactory and other sensory processes during prolonged olfactory stimuli, similar to fluctuations of perception seen during bistable ambiguous visual stimuli (for a review, see Long \& Toppino, 2004).

Our data reveal that odor desensitization demonstrate the presence of low-frequency changes in the focused-attention condition. When attention was focused toward odor, a regular pattern of odor detection appears to recur at between 1 and 2minute intervals following the initial desensitization. This observation was confirmed by significantly greater spectral power in the $0.008-0.015 \mathrm{~Hz}$ range in the focused-attention, relative to distraction, condition. We conjecture that olfactory desensitization is a dynamic process, whereby attentional factors may modulate switching between odor-related percepts. Previously, attention has been shown to modulate fluctuations between perceptions of ambiguous visual stimuli (Leopold \& Logothetis, 1999; Long \& Toppino, 2004), or rival binocular and binaural stimuli (Blake \& Logothetis, 2002; Brancucci \& Tommasi, 2011; Paffen, Alais, \& Verstraten, 2006). Therefore, it is likely that focused attention can also modulate the process by which the olfactory environment is continuously reevaluated during a prolonged stimulus. Additional factors could also play a relevant role in recurring perception during a dual-task process. Previous research suggests that working memory capacity could modulate orientation response to cross-modal (auditory) stimuli (Sörqvist, Nöstl, \& Halin, 2012), and the role of increased cognitive load (e.g., occurring in the distraction condition) also warrants further research. 
Previous studies have manipulated the context in which an odor may be perceived, and this process is likely to influence the degree of attention allocated toward an odor (Dalton, 1996). However, the primary focus of attention in such studies was always directed toward the odor. Attention for olfaction is a selective process (Keller, 2011), and the present study employed a deliberate attentional manipulation to clearly focus attention toward, or distract it away from, the odor with no manipulation of context. This type of manipulation was previously utilized to demonstrate neurophysiological effects of focused attention on odor perception using EEG (Krauel et al., 1998), and behavioral studies also demonstrated a significant effect of competing multimodal stimuli on odor perception (Spence et al., 2000; Spence, Kettenmann, et al., 2001; Spence, McGlone, et al., 2001). However, the method has, to our knowledge, never been utilized to investigate the role of attention for ongoing desensitization to prolonged stimuli. As such, the present study represents the first direct evidence that allocation of attention modulates desensitization to prolonged odor stimuli and ongoing perception of the olfactory environment, irrespective of other top-down processes. One fMRI study previously demonstrated increased cortical activations across a period of desensitization in ignore, relative to focused attention, conditions (Sabri et al., 2005). However, this research utilized detection of occurrences of deviant olfactory stimuli that differs from the present task. The contrasting findings in our study point to enhanced perception in focused attention conditions, and this difference may indicate specific mechanisms that modulate the relationship between attention and olfaction during consistent stimuli compared with novel or deviant occurrences.

The analysis of respiratory patterns indicates that the results of the present study are not attributable to differences in the rate of respiration between conditions, while the withinsubjects design minimizes the influence of other physiological factors. The finding of significantly closer correlations between odor ratings and respiration rate in distracted, relative to focused, attention suggest that perception tends to follow respiration rate more closely when we are distracted. We may conjecture that this points to an increased likelihood of central factors governing the enhanced perception ratings seen in focused conditions, although further research (e.g., utilizing neuroimaging techniques) is needed to confirm this.

The relatively small number of participants should be considered as a limitation of the present design, and further research is needed to replicate and expand on the present findings. Likewise, the odor utilized in the present study exhibited little or no trigeminal profile, but future studies should compare the role of trigeminal, relative to purely olfactory, contributions for recurring odor perception during desensitization. For curve fitting, we opted for a group-level method based upon classical analyses of human olfactory stimulus-response curves (Chastrette, Thomas-Danguin, \& Rallet, 1998). An alternative, even preferable, method would have been to evaluate individual response curves prior to group-level tests (Sinding et al., 2017). However, this method was not selected due to the reduced signal-to-noise inherent in single prolonged exposures which is a limitation of the current study design.

In conclusion, our findings show modulation of odor desensitization by focus of attention for the first time, exemplifying the importance of top-down control of odor perception. Distraction appears to increase the rate at which desensitization occurs in the initial stages of odor exposure, but also reduces ongoing recurrent sensitivity to odor. Further research is required to investigate the precise time course of this latter phenomenon in particular, for example, to evaluate whether the effect is stable across populations, or odors of differing hedonics or intensities.

Acknowledgements This study was supported by Unilever Ltd.

Open Access This article is distributed under the terms of the Creative Commons Attribution 4.0 International License (http:// creativecommons.org/licenses/by/4.0/), which permits unrestricted use, distribution, and reproduction in any medium, provided you give appropriate credit to the original author(s) and the source, provide a link to the Creative Commons license, and indicate if changes were made.

\section{References}

Berglund, U. (1974). Dynamic properties of the olfactory system. Annals of the New York Academy of Sciences, 237, 17-27.

Blake, R., \& Logothetis, N. (2002). Visual competition. Nature Reviews Neuroscience, 3(1), 13-21. https://doi.org/10.1038/nrn701

Brancucci, A., \& Tommasi, L. (2011). "Binaural rivalry": Dichotic listening as a tool for the investigation of the neural correlate of consciousness. Brain and Cognition, 76(2), 218-224. https://doi.org/10. 1016/j.bandc.2011.02.007

Cain, W. S. (1969). Odor intensity: Differences in exponent of psychophysical function. Perception \& Psychophysics, 6(6), 349-354. https://doi.org/10.3758/Bf03212789

Chastrette, M., Thomas-Danguin, T., \& Rallet, E. (1998). Modelling the human olfactory stimulus-response function. Chemical Senses, 23(2), 181-196.

Dalton, P. (1996). Odor perception and beliefs about risk. Chemical Senses, 21(4), 447-458.

Dalton, P. (2000). Psychophysical and behavioral characteristics of olfactory adaptation. Chemical Senses, 25(4), 487-492. https://doi.org/ 10.1093/chemse/25.4.487

Dalton, P., Wysocki, C. J., Brody, M. J., \& Lawley, H. J. (1997). The influence of cognitive bias on the perceived odor, irritation and health symptoms from chemical exposure. International Archives of Occupational and Environmental Health, 69(6), 407-417. https://doi.org/10.1007/s004200050168

Ekman, G., Berglund, B., Berglund, U., \& Lindvall, T. (1967). Perceived intensity of odor as a function of time of adaptation. Scandinavian Journal of Psychology, 8(3), 177-186. https://doi.org/10.1111/j. 1467-9450.1967.tb01392.x

Geisler, M. W., \& Murphy, C. (2000). Event-related brain potentials to attended and ignored olfactory and trigeminal stimuli. International Journal of Psychophysiology, 37(3), 309-315. 
Hoffmann-Hensel, S. M., Sijben, R., Rodriguez-Raecke, R., \& Freiherr, J. (2017). Cognitive load alters neuronal processing of food odors. Chemical Senses, 42(9), 723-736. https://doi.org/10.1093/chemse/ bjx046

Hummel, T., Knecht, M., \& Kobal, G. (1996). Peripherally obtained electrophysiological responses to olfactory stimulation in man: Electro-olfactograms exhibit a smaller degree of desensitization compared with subjective intensity estimates. Brain Research, $717(1 / 2), 160-164$

Hummel, T., Sekinger, B., Wolf, S. R., Pauli, E., \& Kobal, G. (1997). 'Sniffin'Sticks': Olfactory performance assessed by the combined testing of odor identification, odor discrimination and olfactory threshold. Chemical Senses, 22(1), 39-52.

Jacob, T. J., Fraser, C., Wang, L., Walker, V., \& O’Connor, S. (2003). Psychophysical evaluation of responses to pleasant and mal-odour stimulation in human subjects: Adaptation, dose response and gender differences. International Journal of Psychophysiology, 48(1), 67-80.

Keller, A. (2011). Attention and olfactory consciousness. Frontiers in Psychology, 2, 380. https://doi.org/10.3389/fpsyg.2011.00380

Kobayashi, T., Sakai, N., Kobayakawa, T., Akiyama, S., Toda, H., \& Saito, S. (2008). Effects of cognitive factors on perceived odor intensity in adaptation/habituation processes: From 2 different odor presentation methods. Chemical Senses, 33(2), 163-171. https:// doi.org/10.1093/chemse/bjm075

Krauel, K., Pause, B. M., Sojka, B., Schott, P., \& Ferstl, R. (1998). Attentional modulation of central odor processing. Chemical Senses, 23(4), 423-432.

Kurahashi, T., \& Menini, A. (1997). Mechanism of odorant adaptation in the olfactory receptor cell. Nature, 385(6618), 725-729. https://doi. org $/ 10.1038 / 385725 \mathrm{a} 0$

Leopold, D. A., \& Logothetis, N. K. (1999). Multistable phenomena: Changing views in perception. Trends in Cognitive Sciences, 3(7), 254-264.

Long, G. M., \& Toppino, T. C. (2004). Enduring interest in perceptual ambiguity: Alternating views of reversible figures. Psychological Bulletom, 130(5), 748-768. https://doi.org/10.1037/0033-2909. 130.5.748

Maris, E., \& Oostenveld, R. (2007). Nonparametric statistical testing of EEG- and MEG-data. Journal of Neuroscience Methods, 164(1), 177-190. https://doi.org/10.1016/j.jneumeth.2007.03.024

Masago, R., Shimomura, Y., Iwanaga, K., \& Katsuura, T. (2001). The effects of hedonic properties of odors and attentional modulation on the olfactory event-related potentials. Journal of Physiological Anthropology and Applied Human Science, 20(1), 7-13.

Paffen, C. L., Alais, D., \& Verstraten, F. A. (2006). Attention speeds binocular rivalry. Psychological Science, 17(9), 752-756. https:// doi.org/10.1111/j.1467-9280.2006.01777.x

Pause, B. M., Sojka, B., \& Ferstl, R. (1997). Central processing of odor concentration is a temporal phenomenon as revealed by chemosensory event-related potentials (CSERP). Chemical Senses, 22(1), 9-26. https://doi.org/10.1093/chemse/22.1.9

Pellegrino, R., Sinding, C., de Wijk, R. A., \& Hummel, T. (2017). Habituation and adaptation to odors in humans. Physiological Behavior, 177, 13-19. https://doi.org/10.1016/j.physbeh.2017.04.006

Plailly, J., Howard, J. D., Gitelman, D. R., \& Gottfried, J. A. (2008). Attention to odor modulates thalamocortical connectivity in the human brain. Journal of Neuroscience, 28(20), 5257-5267. https://doi. org/10.1523/JNEUROSCI.5607-07.2008

Poellinger, A., Thomas, R., Lio, P., Lee, A., Makris, N., Rosen, B. R., \& Kwong, K. K. (2001). Activation and habituation in olfaction-An fMRI study. NeuroImage, 13(4), 547-560. https://doi.org/10.1006/ nimg. 2000.0713
Rolls, E. T., Grabenhorst, F., Margot, C., da Silva, M. A., \& Velazco, M. I. (2008). Selective attention to affective value alters how the brain processes olfactory stimuli. Journal of Cognitive Neuroscience, 20(10), 1815-1826. https://doi.org/10.1162/jocn.2008.20128

Sabri, M., Radnovich, A. J., Li, T. Q., \& Kareken, D. A. (2005). Neural correlates of olfactory change detection. NeuroImage, 25(3), 969974. https://doi.org/10.1016/j.neuroimage.2004.12.033

Sinding, C., Valadier, F., Al-Hassani, V., Feron, G., Tromelin, A., Kontaris, I., \& Hummel, T. (2017). New determinants of olfactory habituation. Scientific Reports, 7, 41047. https://doi.org/10.1038/ srep41047

Smeets, M., \& Dalton, P. (2002). Perceived odor and irritation of isopropanol: A comparison between naive controls and occupationally exposed workers. International Archives of Occupational and Environmental Health, 75(8), 541-548. https://doi.org/10.1007/ s00420-002-0364-y

Smeets, M. A. M., \& Dalton, P. H. (2005). Evaluating the human response to chemicals: Odor, irritation and non-sensory factors. Environmental Toxicology and Pharmacology, 19(3), 581-588. https://doi.org/10.1016/j.etap.2004.12.023

Sobel, N., Prabhakaran, V., Zhao, Z., Desmond, J. E., Glover, G. H., Sullivan, E. V., \& Gabrieli, J. D. (2000). Time course of odorantinduced activation in the human primary olfactory cortex. Journal of Neurophysiology, 83(1), 537-551.

Sörqvist, P., Nöstl, A., \& Halin, N. (2012). Working memory capacity modulates habituation rate: Evidence from a cross-modal auditory distraction paradigm. Psychonomic Bulletin \& Review, 19(2), 245250. https://doi.org/10.3758/s13423-011-0203-9

Spence, C., Kettenmann, B., Kobal, G., \& McGlone, F. P. (2000). Selective attention to the chemosensory modality. Perception \& Psychophysics, 62(6), 1265-1271. https://doi.org/10.3758/ Bf03212128

Spence, C., Kettenmann, B., Kobal, G., \& McGlone, F. P. (2001). Shared attentional resources for processing visual and chemosensory information. Quarterly Journal of Experimental Psychology Section AHuman Experimental Psychology, 54(3), 775-783. https://doi.org/ 10.1080/02724980042000480

Spence, C., McGlone, F. P., Kettenmann, B., \& Kobal, G. (2001). Attention to olfaction-A psychophysical investigation. Experimental Brain Research, 138(4), 432-437. https://doi.org/10. $1007 / \mathrm{s} 002210100713$

Stuck, B. A., Fadel, V., Hummel, T., \& Sommer, J. U. (2014). Subjective olfactory desensitization and recovery in humans. Chemical Senses, 39(2), 151-157. https://doi.org/10.1093/chemse/bjt064

Thompson, R. F., \& Spencer, W. A. (1966). Habituation: A model phenomenon for the study of neuronal substrates of behavior. Psychological Review, 73(1), 16-43.

Veldhuizen, M. G., \& Small, D. M. (2011). Modality-specific neural effects of selective attention to taste and odor. Chemical Senses, 36(8), 747-760. https://doi.org/10.1093/chemse/bjr043

Wilson, D. A., \& Sullivan, R. M. (2011). Cortical processing of odor objects. Neuron, 72(4), 506-519. https://doi.org/10.1016/j.neuron. 2011.10.027

Wysocki, C. J., Dalton, P., Brody, M. J., \& Lawley, H. J. (1997). Acetone odor and irritation thresholds obtained from acetone-exposed factory workers and from control (occupationally unexposed) subjects. American Industrial Hygiene Association Journal, 58(10), 704-712. https://doi.org/10.1202/0002-8894(1997)058<0704:Aoaito >2.0.Co;2

Zelano, C., Bensafi, M., Porter, J., Mainland, J., Johnson, B., Bremner, E., ... Sobel, N. (2005). Attentional modulation in human primary olfactory cortex. Nature Neuroscience, 8(1), 114-120. https://doi.org/ $10.1038 / \mathrm{nn} 1368$ 